Int. J. Morphol.,

35(2):515-519, 2017

\title{
Foramen Meningo Orbitario
}

\author{
Meningo Orbital Foramen
}

Juan Silva'; Claudia Araya ${ }^{1}$; Eliza Lagos ${ }^{1}$; Andrés Salcedo ${ }^{1}$ \& Alexis Latín ${ }^{1}$

SILVA, J.; ARAYA, C.; LAGOS, E. ; SALCEDO, A. \& LATÍN, A. Foramen meningo orbitario. Int. J. Morphol., 35(2):515-519, 2017.

RESUMEN: El foramen meningo orbitario (FMO), ubicado en el ala mayor del esfenoides y cercano al extremo lateral de la fisura orbitaria superior (FOS), comunica órbita con fosa craneal media, permitiendo el paso de una anastomosis entre las arterias oftálmica y meníngea media. Su prevalencia varía del 6 al 82,9 \% y puede presentarse en forma unilateral o bilateral, único o múltiple y de forma circular, ovoidal o de ranura. Nuestro objetivo fue evidenciar la presencia del FMO, describiendo sus características morfológicas, en cráneos secos de individuos adultos chilenos de ambos sexos. Se utilizaron 54 cráneos con ambas órbitas. Se consideró; presencia, unilateralidad o bilateralidad, cantidad de forámenes por órbita, forma, ubicación en relación al plano horizontal determinado por el extremo lateral de la FOS, distancia al extremo lateral de la FOS, diámetro y diferencias sexuales. Las mediciones se realizaron con sonda de nylon, compás de puntas secas y cáliper digital. Un 46,29 \% de la muestra presentó FMO, con mayor presencia en individuos femeninos. En un $28 \%$ se presentó unilateral y en un $72 \%$ bilateral. Predominó la forma circular $(79,07 \%)$ sobre la ovoidal (18 \%) y sobre la forma de ranura $(2,33 \%)$. El FMO se observó en un 90,69 \% sobre el plano horizontal que determina el extremo lateral de la FOS y en un 9,31\% en el mismo plano. La distancia del FMO al extremo lateral de la FOS fue de 6,58 mm y el diámetro del FMO correspondió a 1,22 mm. Nuestros resultados coinciden con la literatura, respecto a su presencia, a la comunicación que permite, a su ubicación, a su forma y tamaño. Se pudo constatar semejanzas y algunas diferencias menores con cráneos indios, asiáticos y pakistaníes. También pudimos evidenciar diferencias por sexo. El conocimiento acabado del FMO tiene importancia en anatomía, antropología, oftalmología, traumatología, imagenología, cirugía e identificación humana.

PALABRAS CLAVE: Órbita; Fisura orbitaria superior; Foramen meningo orbitario; Arteria oftálmica; Arteria meníngea media.

\section{INTRODUCCIÓN}

En el extremo lateral de la fisura orbitaria superior (FOS), se presenta una incisura, convertida algunas veces en un foramen, para el paso de un ramo recurrente de la arteria oftálmica destinado a la duramadre (Cruveilhier, 1851). Este foramen se denomina foramen meningo orbitario (FMO) (Fig. 1) y es un foramen propio de la órbita que permite la comunicación entre esta cavidad y la fosa craneal media, y representa un conducto embrionario entre la división supraorbitaria de la arteria estapedial y una rama de la arteria oftálmica (Georgiou \& Casell, 1992; Aziz \& Malik, 1997; Jhadav et al., 2012; Celik et al., 2014; Agarwall et al., 2015; Garapati et al., 2016). También se describe que en algunos casos, permitiría la comunicación entre órbita y fosa craneal anterior (Jhadav et al., 2012; Agarwall et al., 2015).

Este foramen permite el paso de una anastomosis entre una rama recurrente meníngea de la arteria oftálmica, o de la arteria lagrimal, con una rama orbitaria de la arteria meníngea media (Wolff, 1954; Testut \& Latarjet, 1965; Goss, 1976; Dilenge \& Ascherl, 1980; Diamond, 1990; Georgiou \& Casell; Agur \& Lee; 1994; Aziz \& Malik; René, 2006; Jhadav et al., 2012; Celik et al.; Agarwall et al.; Macchi et al., 2016). Este vaso comunicante representa una anastomosis entre las arterias carótidas interna y externa (Wolff), y en forma normal esta arteria anstomótica cruza por la FOS (Lockhart et al., 1998). Este FMO también puede permitir el paso de la arteria meníngea media oftálmica, variante anatómica de la arteria meníngea media como rama de la arteria oftálmica (Manjunath, 2001).

El FMO se describe como un foramen ubicado en el ala mayor del esfenoides (Georgiou \& Casell; Aziz \& Malik; Jhadav et al., 2012; Agarwall et al.), cercano al extremo lateral de la FOS (Testut \& Latarjet; Agur \& Lee) y en algunos casos se puede observar confluir con esta fisura (Jhadav et al., 2012) o solo presentar la forma de una ranura en el espesor de la sutura fronto-esfenoidal (René). 
La prevalencia del FMO descrita en la literatura varía del 6 al 82,9\% en cráneos de distintos orígenes. Además este foramen se puede presentar único o múltiple y de forma unilateral o bilateral (Georgiou \& Casell; Aziz \& Malik; Jhadav et al., 2012; Celik et al.; Agarwall et al.; Garapati et al.; Macchi et al.).

El conocimiento de las variaciones anatómicas de los forámenes del cráneo es importante en anatomía, antropología, oftalmología, cirugía, traumatología, imagenología e identificación humana.

Nuestro objetivo principal fue evidenciar la presencia del FMO y describir sus características morfológicas fundamentales en cráneos secos de individuos adultos chilenos de ambos sexos.

\section{MATERIAL Y MÉTODO}

Se analizaron 54 cráneos de individuos adultos chilenos, 27 de sexo masculino y 27 de sexo femenino, pertenecientes a la osteoteca de la Escuela de Medicina de la Universidad San Sebastián, en búsqueda de FMO. Estos cráneos debían presentar ambas órbitas.

Para considerar la presencia del FMO, éste debía ser permeable a una sonda de nylon de $0,25 \mathrm{~mm}$ de espesor y comunicar órbita con la fosa craneal media o con la fosa craneal anterior.

De los FMO encontrados, se consideró su presencia (unilateral o bilateral), la cantidad de forámenes por órbita (únicos o múltiples), su forma (circular, ovoidal o ranura), su ubicación en relación al plano horizontal determinado por el extremo lateral de la FOS (por encima del plano, en el mismo plano o debajo de este plano), su distancia del extremo lateral de la FOS, la dimensión de su diámetro mayor y sus diferencias sexuales. Todos estos datos se registraron en planilla ad hoc para su análisis.

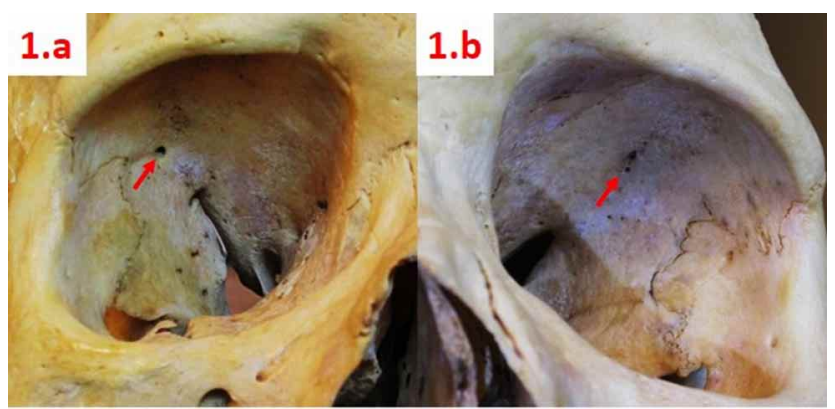

Fig. 1a) FMO en órbita derecha. b) FMO en órbita izquierda.
Todas las mediciones se realizaron con compas de puntas secas y caliper digital marca Mitutoyo $^{\circ}$ serie 500 modelo 144.

Los cráneos se fotografiaron con cámara digital marca Canon $^{\oplus}$ modelo PowerShot A3500 IS.

\section{RESULTADOS}

Del total de la muestra (54 cráneos), un 46,29\% presentó FMO (25 cráneos). De los 27 cráneos de individuos femeninos, un 55,55 \% presentó FMO (15 cráneos) y de los 27 cráneos de individuos masculinos, un 37,03\% presentó FMO (10 cráneos).

De los 25 cráneos que presentaron FMO, 7 de ellos (28\%) se observaron en solo una de sus órbitas (unilaterales), mientras que 18 (72\%) presentaron en ambas (bilaterales) (Fig. 2).

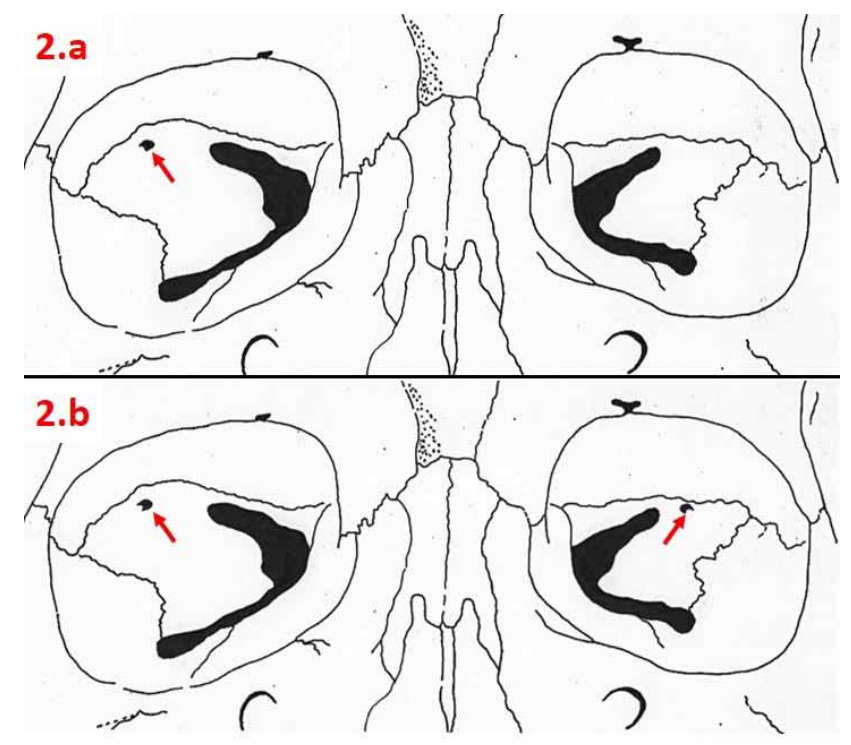

Fig. 2a) FMO unilateral (flecha). b) FMO bilateral (flechas).

En los FMO encontrados, primó la forma circular en un 79,07\% de los casos, seguida por la forma ovoidal en un $18,60 \%$. Mientras que la forma de ranura (en la sutura frontoesfenoidal) se observó en un 2,33 \% de los casos (Fig. 3).

De la ubicación del FMO se pudo observar que en un $90,69 \%$ de la muestra, el foramen se disponía sobre el plano horizontal que determina el extremo lateral de la FOS y en un 9,31\% se disponía en el mismo plano. No fue posible observar FMO bajo este plano (Fig. 4a). 


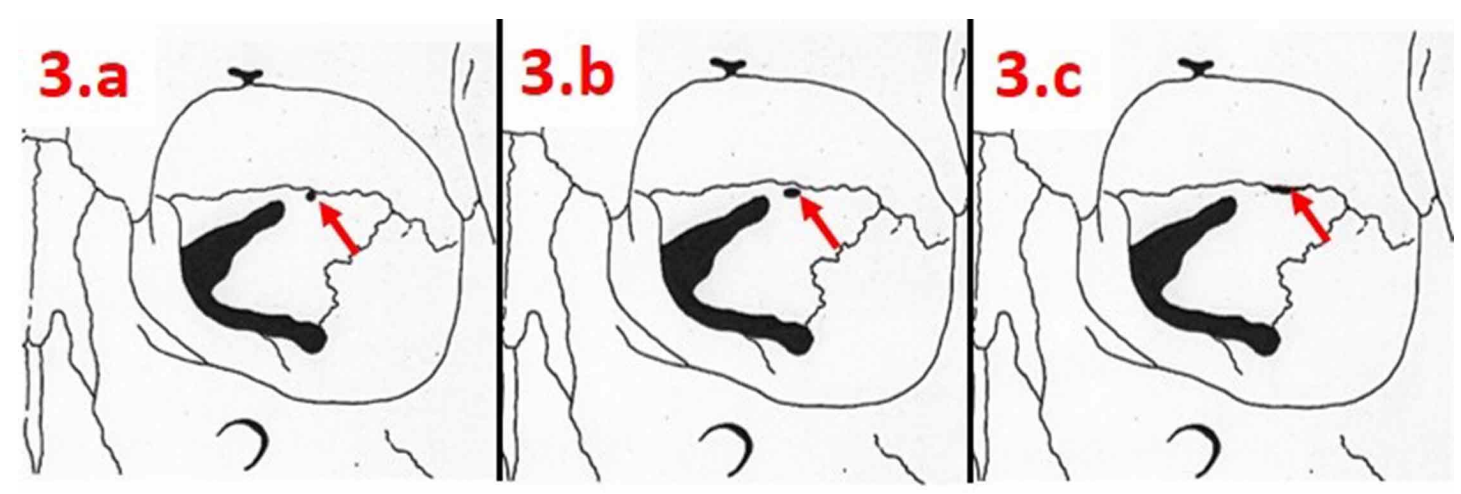

Fig. 3. a) FMO de forma circular (flecha). b) FMO de forma ovoidal (flecha). c) FMO en forma de ranura (flecha).

La distancia del FMO al extremo lateral de la FOS (Fig. 4b) en promedio, midió $6,58 \mathrm{~mm}$, (rango de $1,36 \mathrm{~mm}$ a $12,10 \mathrm{~mm}$ ). Esta distancia en las órbitas derechas fue de $6,27 \mathrm{~mm}$ en promedio, (rango de $1,36 \mathrm{~mm}$ a $10,98 \mathrm{~mm}$ ) y en la órbita izquierda el promedio de la citada distancia fue de $6,86 \mathrm{~mm}$ (rango de 2,20 $\mathrm{mm}$ a 12,10 $\mathrm{mm}$ ).

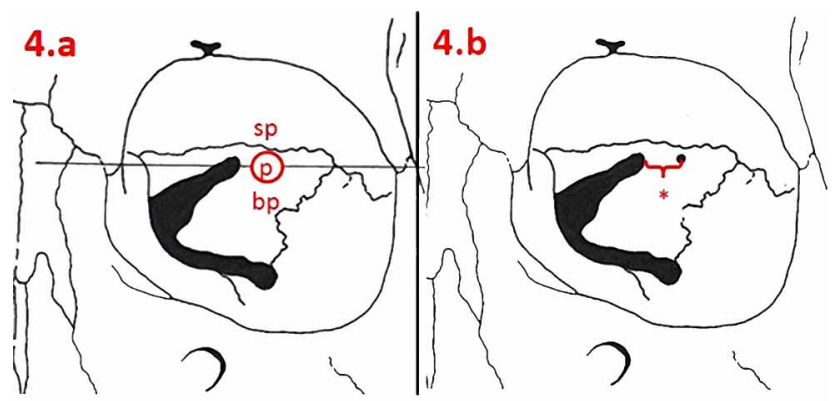

Fig. 4. a) Relación del FMO con el plano horizontal determinado por el extremo lateral de la FOS (sp: sobre el plano, p: en el plano, bp: bajo el plano). b) Distancia del FMO al extremo lateral de la FOS (asterisco).

La medida promedio del diámetro mayor del FMO correspondió a 1,22 $\mathrm{mm}$ (rango de 0,26 mm a 1,96 mm). En las órbitas derechas este diámetro se determinó, en promedio, en $1,21 \mathrm{~mm}$ (rango de $0,26 \mathrm{~mm}$ a $1,84 \mathrm{~mm}$ ), mientras que en la izquierda este diámetro fue de $1,22 \mathrm{~mm}$ (rango de los $0,6 \mathrm{~mm}$ y los $1,96 \mathrm{~mm}$ ).

En los cráneos de individuos femeninos con FMO bilateral ( $80 \%$ ), se pudo observar la siguiente distribución: 4 presentaron forámenes únicos $(26,66 \%), 3$ presentaron forámenes múltiples ( $20 \%), 2$ presentaron foramen múltiple en la órbita derecha y foramen único en la izquierda $(13,33$ $\%), 3$ presentaron foramen único en la órbita derecha y forámenes múltiples en la izquierda (13,33\%). En los individuos con FMO unilateral (20 \%), 1 cráneo presentó foramen múltiple en la órbita derecha $(6,66 \%)$ y 2 cráneos presentaron foramen único en la órbita izquierda (13,33\%) (Fig. 5).

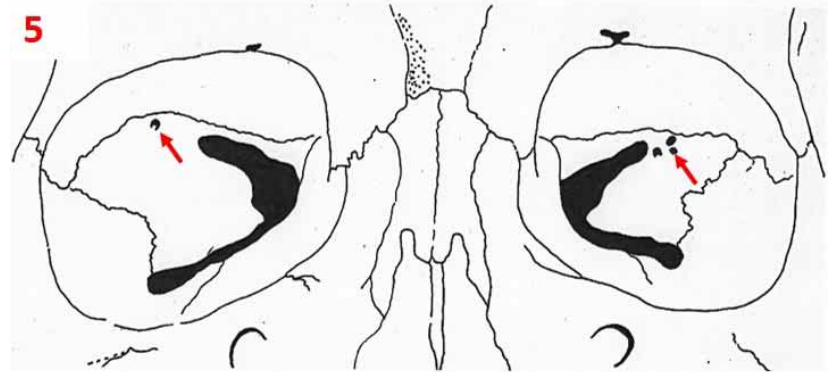

Fig. 5. En órbita derecha, FMO único (flecha). En órbita izquierda, FMO múltiple (flecha).

En los cráneos de individuos femeninos primó la forma circular en un $85,19 \%$ de los casos, seguida por la forma ovoidal en un $11,11 \%$ de los casos. Mientras que la forma de ranura (en la sutura fronto-esfenoidal) se observó en un $3,70 \%$ de los casos.

De la ubicación del FMO en cráneos de individuos femeninos, se pudo observar que un $88,88 \%$ de la muestra el foramen se disponía sobre el plano horizontal que determina el extremo lateral de la FOS y en un $11,11 \%$ se dispone en el mismo plano.

En estos mismos cráneos, la distancia del FMO al extremo lateral de la FOS en promedio midió 6,20 mm (rango de $1,36 \mathrm{~mm}$ a $11,87 \mathrm{~mm}$ ). Esta distancia en las órbitas derechas fue de 6,01 $\mathrm{mm}$ en promedio (rango entre 1,36 $\mathrm{mm}$ a $10,98 \mathrm{~mm}$ ).

En la órbita izquierda el promedio de la citada distancia fue de 6,39 $\mathrm{mm}$ (rango de 2,20 $\mathrm{mm}$ a $11,87 \mathrm{~mm}$ ).

En estos mismos cráneos, la medida promedio del diámetro mayor del FMO correspondió a un 1,19 mm (rango de $0,26 \mathrm{~mm}$ a $1,96 \mathrm{~mm}$ ). En las órbitas derechas se pudo determinar un promedio de $1,17 \mathrm{~mm}$ (rango de $0,26 \mathrm{~mm}$ a $1,80 \mathrm{~mm}$ ), mientras que en la izquierda su promedio fue de $1,21 \mathrm{~mm}$ (rango de $0,6 \mathrm{~mm}$ a $1,96 \mathrm{~mm}$ ). 
En los cráneos de individuos masculinos con FMO bilateral (60\%), se pudo observar la siguiente distribución: 1 presentó forámenes únicos $(10 \%), 4$ presentaron forámenes múltiples $(40 \%)$ y 1 presentó foramen único en la órbita derecha y foramen múltiple en la izquierda (10\%). Mientras que en los individuos con FMO unilateral (40\%), 2 individuos presentaron foramen múltiple en la órbita derecha $(20 \%)$ y 2 individuos presentaron foramen único en la órbita izquierda $(20 \%)$.

En los cráneos de individuos masculinos primó la forma circular en un $68,75 \%$ de los casos, seguido por la forma ovoidal en un $31,25 \%$. No se pudo evidenciar la forma de ranura.

De la ubicación del FMO en los cráneos de individuos masculinos, se pudo observar que un $93,75 \%$ de la muestra, el foramen se disponía sobre el plano horizontal que determina el extremo lateral de la FOS y en un 6,25\% se dispone en el mismo plano.

En estos mismos cráneos, la distancia del FMO al extremo lateral de la FOS midió 7,21 mm en promedio (rango de $1,89 \mathrm{~mm}$ a $12,01 \mathrm{~mm}$ ). Esta distancia en las órbitas derechas fue de 6,71 $\mathrm{mm}$ en promedio (rango de 1,89 $\mathrm{mm}$ a $9,69 \mathrm{~mm}$ ). En la órbita izquierda el promedio en la citada distancia fue de 7,7 $\mathrm{mm}$ (rango de 3,68 $\mathrm{mm}$ a 12,01 $\mathrm{mm}$ ).

Por otra parte, la medida promedio del diámetro mayor del FMO correspondió a un 1,26 mm (rango de 0,83 $\mathrm{mm}$ a $1,84 \mathrm{~mm}$ ). En las órbitas derechas se pudo determinar un promedio de $1,29 \mathrm{~mm}$ (rango de $0,83 \mathrm{~mm}$ a $1,84 \mathrm{~mm}$ ), mientras que en la izquierda su promedio fue de $1,24 \mathrm{~mm}$ (rango de $0,84 \mathrm{~mm}$ a $1,56 \mathrm{~mm}$ ).

\section{DISCUSIÓN}

Nuestra investigación respecto a la existencia del FMO, es bastante coincidente con lo reportado en la literatura, pudiéndose evidenciar su presencia en la cara orbitaria del ala mayor del hueso esfenoides y lateral al extremo lateral de la FOS, comunicando órbita con fosa craneal media (Georgiou \& Cassell; Agur \& Lee; Aziz \& Malik; Jadhav et al.; Celik et al.; Agarwall et al.; Garapati et al.; Macchi et al.).

La prevalencia del FMO sin diferenciar por sexo, fue de un 46,29\%, semejante a lo reportado en cráneos de individuos de la India (Jhadav, et al.; Agarwall et al.; Garapati et al.). También los resultados son semejantes a lo observado en cráneos asiáticos (Georgiou \& Cassell), pero es menor al $66 \%$ descrito en cráneos pakistaníes (Aziz \& Malik). Además, es menor a lo reportado por Macchi et al. $(59,46 \%)$.

Nuestros resultados mostraron que en cráneos de individuos de sexo femenino, el porcentaje de FMO $(55,55$ $\%$ ) fue inferior al $58 \%$ observado en cráneos indios (Jadhav et al.), pero significativamente superior al $41,14 \%$ encontrado por Macchi et al. En los cráneos de individuos masculinos, la presencia de FMO ( 37,03 \%), es semejante al 37,5 $\%$ encontrado en cráneos indios (Jhadav et al.) pero menor al 42,57\% informado por Macchi et al.

Con respecto a la presencia unilateral o bilateral de FMO, pudimos observar que un $28 \%$ era unilateral, ser semejante a lo encontrado en población india (Agarwall et al.; Garapati et al.), pero bastante distinto al $74 \%$ reportado en cráneos de individuos pakistaníes (Aziz \& Malik). La presencia bilateral de FMO en nuestra muestra (72\%), resultó muy por encima de lo encontrado tanto en cráneos indios (Agarwall et al.; Garapati et al.) como en cráneos pakistaníes (Aziz \& Malik).

En relación a la forma de la FMO, casi el $80 \%$ de forma circular y solamente un $18,6 \%$ forma ovoidal, coincide con lo encontrado en cráneos indios (Jhadav et al.). En muy pocos cráneos (alerdedor del $2 \%$ ) presentaron la forma de ranura en el espesor de la sutura fronto esfenoidal, coincidiendo con lo descrito por René.

La ubicación de la FMO, se presentó aproximadamente en el $90 \%$ sobre el plano horizontal que marca el extremo lateral de la FOS, mientras que menos del $10 \%$ de los cráneos mostró el MFO en el plano, no pudiendo observar FMO debajo de ese mismo plano. Estos resultados son acorde a lo descrito por Georgiou \& Cassell.

También resultó coincidente con los datos reportados por Agarwall et al. la distancia de la FMO al extremo de la FOS, sin considerar diferencias por sexo $(6,58 \mathrm{~mm})$, en mujeres $(6,2 \mathrm{~mm})$ y en hombres $(7,21 \mathrm{~mm})$.

Por otra parte, el diámetro promedio del FMO sin diferenciar sexo o lado, correspondió a 1,22 mm. Estos resultados son relativamente concordantes con lo indicado por Macchi et al.

En aquellos individuos que presentaron FOM bilaterales, ya sean únicos, múltiples en ambos lados, múltiples en órbita derecha - foramen único en órbita izquierda y foramen único en órbita derecha - foramen múltiple en izquierda, resultan ser discordantes en los repórteres de Aziz \& Malik en cráneos pakistaníes. 
En conclusión, indicaremos que el conocimiento acabado del FMO tiene importancia, desde el punto de vista disciplinar anatómico y antropológico (el estudio muestra semejanzas y diferencias étnicas). También es de vital importancia para el diagnóstico y terapéutica en oftalmología y en traumatología, para la correcta interpretación imgenológica de las características anatómicas de la órbita y de la base del cráneo, para la planificación del operatorio y postoperatorio en procedimientos quirúrgicos y para la identificación humana en medicina legal.

SILVA, J.; ARAYA, C.; LAGOS, E. ; SALCEDO, A. \& LATÍN, A. Meningo Orbital Foramen. Int. J. Morphol., 35(2):500-505, 2017.

SUMMARY : The meningo orbital foramen (MOF) is located in the major wing of the sphenoid and near the lateral end of the superior orbital fissure (SOF), communicating orbit with the middle cranial fossa and allowing the passage of an anastomosis between the ophthalmic and middle meningeal arteries. Its prevalence varies from 6 to $82.9 \%$ and may occur unilaterally or bilaterally, single or multiple, and may have a circular, ovoid or groove form. The aim of this study is to evidence the existence of MOF, describing its morphological characteristics in dry skulls of Chilean adults of both sexes. The present study analyzed 54 skulls containing both orbits, considering the following criteria: Existence, unilaterality or bilaterality, number of foramens by orbit, shape, location in relation to the horizontal baseline determined by the lateral end of the SOF, distance to the lateral end of the SOF and diameter and variations by sex. Measurements were made with nylon probe, compass dry point and digital caliper. Of the sample, $46.29 \%$ presented MOF with a greater prevalence in female samples. In $28 \%$ of the cases it was unilateral and in the other $72 \%$ bilateral. The circular shape was predominant in $79.07 \%$, followed by the ovoidal $18 \%$ and the groove form $2.33 \%$. The MOF was observed in $90.69 \%$ on the horizontal baseline that determines the lateral end of the SOF and in $9.31 \%$ in the same baseline. The average distance from the MOF to the lateral end of the SOF was $6.58 \mathrm{~mm}$ and the diameter of the MOF corresponded to $1.22 \mathrm{~mm}$. Our results are consistent with similar studies. There were some minor differences observed in Indian, Asian and Pakistani skulls as well as some anatomical differences by sex. A better understanding of MOF plays an important role in anatomy, anthropology, ophthalmology, traumatology, medical imaging, surgery and human identification.

KEY WORDS: Orbit; Superior orbital fissure; Meningo orbital foramen; Ophthalmic artery; Middle meningeal artery.

\section{REFERENCIAS BIBLIOGRÁFICAS}

Agarwall, C.; Garg, R.; Kumar, S.; Sharma, D. \& Pareek, P. Foramen meningo-orbitale: its incidence and clinical significance in Indians. Indian J. Basic Appl. Med. Res., 4(4):127-32. 2015.

Agur, A. M. R. \& Lee, M. J. Grant Atlas de Anatomía. 9ª ed. Buenos Aires, Editorial Medica Panamericana, 1994.

Aziz, N. \& Malik, Y. Incidence of foramen meningo-orbitale in adult Pakistani skulls. Proc. S. Z. P. G. M. I., 11(3-4):52-5, 1997.

Celik, S.; Kazak, Z.; Ozer, M. A. \& Govsa, F. Navigational area of the cranio-orbital foramen and its significance in orbital surgery. Surg. Radiol. Anat., 36(10):981-8, 2014.

Cruveilhier, J. Tratado de Anatomía Descriptiva. Madrid, Bailly-Bailliére, 1851.

Diamond, M. K. The groove in the orbital face of the greater wing of the sphenoid. A new interpretation. J. Anat., 173:97-9, 1990.

Dilenge, D. \& Ascherl, G. F. Jr. Variations of the ophthalmic and middle meningeal arteries: relation to the embryonic stapedial artery. A. J. N. R. Am. J. Neuroradiol., 1(1):45-54, 1980.

Garapati, S.; Santhi, Ch. \& Sharmila Bhanu, P. Incidence and clinical significance of cranio-orbital foramen in Telangana Region of South India. Int. J. Anat. Res., 4(2):2297-300, 2016.

Georgiou, C. \& Cassell, M. D. The foramen meningo-orbitale and its relationship to the development of the ophthalmic artery. J. Anat., 180(Pt. 1):119-25, 1992.

Goss, C. M. Gray Anatomía. Barcelona, Salvat, 1976.

Jadhav, S. D.; Roy, P. P.; Ambali, M. P.; Patil, R. J.; Doshi, M. \& Desai, R. The foramen meningo-orbital in Indian dry skulls. N. J. I. R. M., 3(4):469, 2012.

Lockhart, R.; Hamilton, G. \& Fyfe, F. Anatomía Humana. México, Editorial Interamericana, 1988.

Macchi, V.; Regoli, M.; Bracco, S.; Nicoletti, C.; Morra, A.; Porzionato,
A.; De Caro, R. \& Bertelli, E. Clinical anatomy of the orbitomeningeal foramina: variational anatomy of the canals connecting the orbit with the cranial cavity. Surg. Radiol. Anat., 38(2):165-77, 2016.

Manjunath, K. Y. Anomalous origin of the middle meningeal artery - A review. J. Anat. Soc. India, 50(2):179-83, 2001.

René, C. Update on orbital anatomy. Eye (Lond.), 20(10):1119-29, 2006.

Testut, L.; Latarjet, A. \& Latarjet, M. Tratado de Anatomía Humana. $9^{\mathrm{a}}$ ed. Barcelona, Salvat, 1965.

Uflacker, R. Atlas de Anatomía Vascular: Un Abordaje Angiográfico. $2^{\mathrm{a}}$ ed. Caracas, Amolca, 2009.

Wolff, E. The Anatomy of the Eye and Orbit. $4^{\mathrm{a}}$ ed. Londres, H. K. Lewis \& Co. Ltd., 1954.

Direccion para correspondencia:

Prof. Dr. Juan Luis Silva R.

Facultad de Medicina

Universidad San Sebastian

Providencia

Santiago

CHILE

Email: juan.silva@uss.cl

Recibido : 23-01-2017

Aceptado: 09-03-2017 